Aus der Universitäts-Hautklinik in Bonn.

(Direktor: Prof. E. Hoffmann.)

\title{
Ueber eine kombinierte Sachs-Georgi-Wassermannsche
} Reaktion.

Erwiderung zu den Bemerkungen von Kafka in Nr. 10 der D. m. W. Von Egon Keining.

Die Demonstration von Kafka im Hamburger Aerztlichen Verein fand nach dem Sitzungsbericht von F. Wohlwill (Hamburg) in der M: m. W. 1921 Nr. 5 S. 155 am 11. Januar 1921 statt. Das in der D. m. W. erscheinende of fizielle Protokoll dieser Sitzung ist bis jetzt noch nicht publiziert worden, sodaß mir ein ausführlicherer Bericht über die Methodik Kafkas zur Zeit noch gänzlich fẹlt ${ }^{1}$ ).

Ich selbst habe ïber meine kombinierte S.G.-Wa.R. zun ersten Male am 16. Oktober 1920 auf der Tagung der Rheinisch.

1) Anmerkung beider Korrektur: Der Bericht vom 11. I. 1921 ist inzwischen in der D. m. W. 1921 Nr. 9 S. 259 erschienen. 
Westfälischen Dermatologen in Düsseldorf ausführliclı berichtet und die. Methode demonstriert (vgl. Derm. Zsclir. 1921 Nr. 4). Dieser Hinweis findet sich bereits in meiner Arbeit in der.D. m. W. 1921 Nr. 6. Wie aus dem Protokoll der Nieder. rheinischen Gesellschaft für Natur- Und Heilkullde, Bonn, in der D. m. W. 1921 Nr. 5 hervorgelit, habe ich am 15. November 1920 auch dort meine Versuchsanoidnung denonstriert. Der in Nr. 6 der D. m. W. von mir erschienene Originalartikel ist bereits vor diesem Termin der Redaktion der D. m. W. eingereicht worden, diese hat meinen Eigenbericht über diesen Vortrag jedoch nicht publiziert, sondern hat, um unnötige Wiederholungen zu vermeiden, sich mit dem Hinweis auf meinen Originalartikel beschränkt. Dadurch ist auch noch eine Reihe anderer Experimente, über die ich am 15. XI. 1920 in Bonn berichtet habe, leider nicht publiziert worden. Ich werde dies jedoch später nachholen.

Nach Klarstellung dieses Sachverhaltes durch mich wird Kafka, wie ich glaube, mirgegenüber keine Prioritätsansprüche mehr geltend machen.

Die Kafka selbst zweifelhaft gewordene Auffassung, „daß die Hemmung der Hämolyse in den positiven Proben die Folgeder schädigenden (absorbierenden) Wirkung der gebildeten Flocken auf das Komplement darstell ", möchte ich auf Grund von Experimenten, über die ich am 15. XI. 1920 in der Niederrheinischen Gesellschaft, Bonn, gesprocheı habe, die aber gleichfalls unveröffentlicht geblieben sind, ablehnen. Gewinnt man nach dem Vorgehen $M$ a ndelbauns (M. m. W. 1920 Nr. 33 und 43) die Euglabulinfraktion des Serums und setzt man sowohl mit der Restflüssigkeit als auch mit den Euglobulinen die kombinierte S.G.-Wa.R. in der Weise an, wie ich sie in der D. $m$. W. beschrieben habe, so beobachtet man folgendes Verhalten (analog den Resultaten $M$ andelbaums): Mit der Restflüssigkeit erhält man spezifische Sachs-Georgi-Resultate, während der zur Wa.R. ergänzte Versuchversagt. Bei der Euglobulinfraktiondes Serums versagt die S.G.R., während hier die Wa.R. auch in meiner kombinierten Anwendung gelingt. Dieser komplizierte Versuch glückte mir uicht imner, jedoch in einer großen Anzahl von Fällen mit mir klinisch und serologisch gut bekantıten Seren, wie mir scheint, einwandfrei; indessen suche ich durch weitere, gleichsinnige Experimente zur Zeit die Richtigkeit dieses Befundes zu stützen.

Unter der Voraussetzung, daß ich obigen Befund weiterhiil regelmäßig erheben kann, etgibt sicl, folgende Saclilage:

Das eine Mal miBlingt die Wa.R. trotz vorhandener spezifischer Flocken, das andere Mal gerät sie bei fehlenden Flockungen.

Eine schädigende (absorbierende) Wirkungder, Flocken ${ }^{\text {"s }}$ auf dic Komplementkomponente aktiver Seғa (hier Meer. schweinchensera) kann ich, entsprechend diesem Ergeb. uis, nicht anuehmen. 